\title{
OPTIMIZED BIOREGENERATIVE SPACE DIET SELECTION WITH CREW CHOICE
}

Carrie Vicens ${ }^{1}$, Carolyn Wang ${ }^{1}$, Ammar Olabi ${ }^{2 *}$, Peter Jackson ${ }^{1}$, Jean Hunter ${ }^{3}$

${ }^{1}$ School of Operations Research and Industrial Engineering, Rhodes Hall, Cornell University, Ithaca NY 14853

${ }^{2}$ Food Science Department, Stocking Hall, Cornell University, Ithaca NY 14853

${ }^{3}$ Biological and Environmental Engineering Department, Riley-Robb Hall, Cornell University, Ithaca NY 14853

\section{*Corresponding Author:}

Ammar Olabi

Stocking Hall

Cornell University

Ithaca NY 14853

Phone: (607) 255-4122 (work) (607) 253-6433 (home)

Fax: (607) 254-4868Ｅ-mail: AAO6@CORNELL.EDU 


\begin{abstract}
Previous studies on optimization of crew diets have not accounted for choice. A diet selection model with crew choice was developed. Scenario analyses were conducted to assess the feasibility and cost of certain crew preferences, such as preferences for numerous-desserts, high-salt, and high-acceptability foods. For comparison purposes, a no-choice and a random-choice scenario were considered. The model was found to be feasible in terms of food variety and overall costs. The numerous-desserts, high-acceptability, and random-choice scenarios all resulted in feasible solutions costing between 13.2 and $17.3 \mathrm{~kg} \mathrm{ESM/person-day.}$ Only the high-sodium scenario yielded an infeasible solution. This occurred when the foods highest in salt content were selected for the crew-choice portion of the diet. This infeasibility can be avoided by limiting the total sodium content in the crew-choice portion of the diet. Cost savings were found by reducing food variety in scenarios where the preference bias strongly affected nutritional content.
\end{abstract}

KEYWORDS: diet, choice, space, optimization, bioregenerative. 
CONTENT SENTENCE: A diet selection model that incorporates crew choice was developed and tested using sensitivity analyses. 


\section{INTRODUCTION}

As technology continues to advance, the possibility of a manned long-term space mission to Mars for exploratory research becomes more feasible. The preparation for such a mission has brought about a new set of issues centered on the ability to provide advanced life support activities including crew safety, performance, and general well being. Proper nutrition and adequate food intake are essential for the success of long-term space missions. Feedback from astronauts has shown the unique and influential role of food on their sense of well being in space travel (2). The importance of food has also been observed in other human experiences that mimic space missions in terms of their confinement levels (24).

Besides technology, cost has been a major obstacle to achieving a final scenario for a Mars mission. The cost of pre-packaged foods for a 14.5 year Mars mission is substantial, requiring a resupply of nearly $7600 \mathrm{~kg}$ of pre-packaged

foods per year for a crew of six astronauts (4). One possibility for the surface phase of the Mars mission is a bioregenerative life support system in which astronauts produce most of their food from crops grown hydroponically (26). The cost of a bioregenerative diet depends on a number of factors, the most important of which are lighting technology, crop productivity and crew labor $(10,18)$. The 
cost of bioregenerative space diets is usually calculated in equivalent system mass (ESM) units $(4,15)$, as it is in this study. Economic studies of the effect of design choices on the cost of components of a bioregenerative life support space diet and the interactions between them are potentially very useful in the planning of a life support system in planetary missions. This work focuses on the cost of crew choice in a bioregenerative space diet.

Ideally, a bioregenerative diet should be palatable, nutritionally adequate, varied, and low in cost. Mixed integer linear programming is a classical optimization tool that can be used to choose a set of foods that satisfies the various constraints at a minimum cost incurred. The methods and constraints for cost-optimized bioregenerative diets have been discussed previously $(12,19)$. These optimizations were designed to achieve at least a minimum average level of palatability (6.5 on a 9 point hedonic scale), to satisfy crew nutritional requirements (19), and to provide a level of variety at minimum cost. While these optimizations created nutritionally sound and cost-effective diets, they did not allow the astronauts any input into the selection of foods in the diet beyond their initial acceptability ratings of the foods. The optimization routine selected all the needed foods. While taking the initial acceptability ratings into account is a necessary and useful step, it does not give the crew the opportunity to select some of their foods on a cyclic basis. Moreover, if given the same inputs and 
constraints, the linear program will always produce the same results and thereby design an identical menu for each planning cycle. In an earlier study, Kamen and Peyram (13) assessed the consumption and preference of three types of repetitive diets: a 6-day preplanned diet, a 3-day self-planned diet, and a 3-day preplanned diet for 24 days. The 6-day preplanned diet and the 3-day self-planned diets appeared to be superior to the 3-day preplanned diet, and there was a benefit to having the subjects plan their own diets. The element of choice becomes even more essential given the smell and taste alterations reported to occur in space missions $(1,20)$ which may result in changes of food preferences (16). A diet planning system capable of adapting to these changes would be of great benefit.

\section{Previous Research}

Many researchers have already attempted similar models, but none with this particular objective $(5,25)$. Sklan and Dariel (22) developed a computerized model based on a mixed-integer linear programming algorithm to create an optimal human diet, but the diet did not account for individual selection, choices, or preferences. Soden and Fletcher (23) developed a linear programming algorithm for hospitals to alter a daily diet chosen by an individual to meet the needed nutritional requirements. The latter research is the closest one among the studies reviewed to this work. 
Foytik (9) used linear programming to design a diet that provided the same nutritional content as the Thrifty Food Plan designed by the USDA but with a lower cost. The resulting selection model could reduce food cost in low-income households if individuals would alter their eating habits to meet nutritional requirements and reduce expenses. Henson (11) utilized linear programming techniques to analyze the effects of nutritional and non-nutritional factors on the cost of an average diet. This sensitivity analysis provided both an estimation of costs for a variety of diet compositions as well as marginal cost curves for alterations in the nutritional content required by dietary guidelines.

Damij (3) developed an interactive software, NOPS (Nutrition Optimization and Planning Software), that used a combination of linear programming and goal programming to obtain a minimum cost diet which satisfies nutritional constraints. This software did not account for individual choice. Sinha (21) used non-linear programming to determine the optimal serving frequencies of food items in a diet while ensuring availability, production, budgetary, and nutritional constraints. The Convenience Food Logistics Model was developed by the U.S. Army Natick Research Development and Engineering Center in conjunction with Information Technology Solutions, Inc. (6) to evaluate the possible benefits of incorporating convenience food products into Navy 
menus. The authors of that study did not account for choice but their model considered food availability which was not considered in the study reported here.

The diet selection model with crew choice discussed in this work provides the astronauts with greater control over their daily diets within the constraints of high acceptability and proper nutrition. This increase in control should ease the transition astronauts must make from a typical American diet to a near-vegetarian cuisine by allowing the astronauts to choose foods they enjoy.

The objectives of this work were: a) to incorporate the element of crew choice into an optimization process in a bioregenerative diet design, b) to demonstrate its feasibility under a random choice scenario, and c) to perform sensitivity/scenario analysis to assess the effect of certain "choice biases" on the feasibility and cost of the diet.

\section{MATERIALS AND METHODS}

The data used in this study was collected in a $\mathrm{PhD}$ project aimed at the optimization of a bioregenerative life-support space diet (17). In order to incorporate crew choice in a diet selection process, the astronaut-chosen foods 
must be differentiated from the model-chosen foods in the selection model. Once the astronaut-chosen foods are selected, the model takes the set of chosen foods (the PRESELECTED set) as input and optimally selects the remaining foods for the 10-day cycle in order to fulfill nutritional, acceptability and number-ofservings constraints. The following assumptions are adopted in the model:

1. $25-30 \%$ of the meals (servings) in a 10-day cycle could be chosen by the astronauts. Hence the remaining $70-75 \%$ would be re-optimized by the selection model to fulfill all constraints.

2. To ensure ample variety, foods chosen by the astronauts cannot be re-chosen by the selection model.

3. Astronauts would choose their foods at the beginning of each 10-day cycle.

4. All foods can be prepared on site.

5. Foodstuff inventory constraints are not considered.

\section{Model}

The diet selection model is defined in terms of the following sets:

set FOOD = set of foods (e.g. Carrot Soup, Tofu Custard Pie, ...) ;

set $\boldsymbol{N U T R}=$ set of nutrients (see Table 1, middle column $)$;

Set PRESELECTED $=$ set of foods chosen by the crew ;

set PLANNED = set of foods from which the linear selection model

could choose; and 
set $\boldsymbol{B R E A K}=$ set of breakfast foods ;

In addition to the sets listed above, the data required as input to the model consists of the following parameters:

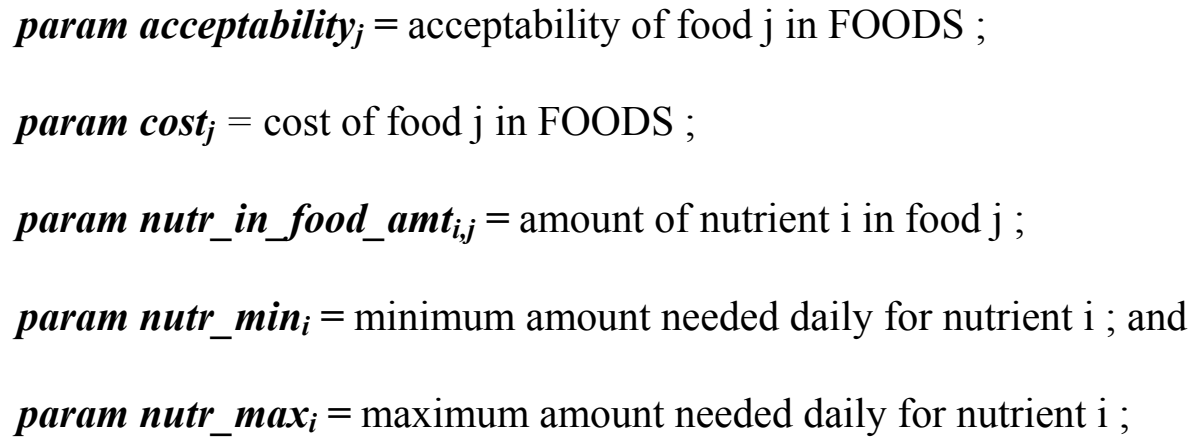

The decision variables of the diet selection model are:

variable $\boldsymbol{x}_{j}=$ number of servings of food $\mathrm{j}$. Food $\mathrm{j}$ could be selected from any of the foods.

The variable $\mathrm{x}_{\mathrm{j}}$, the number of servings of food $\mathrm{j}$, is set to be between 0 and 1 for non-breakfast foods. This restriction ensures that the linear selection model does not choose more than one serving of a certain dish. However, the number of servings of breakfast foods was set to a maximum of 2 . Since there were only a small number of breakfast foods in the study, the model is allowed to choose a breakfast food more than once. In a similar manner, at most one serving is allowed for non-breakfast foods and two servings for breakfast foods for the foods in the choice set (PRESELECTED). Hence the percentage of crew-chosen 
foods varied between $25-30 \%$ in this study depending on the number of breakfast foods in the choice set.

The crew-chosen foods are input to the selection model through the set PRESELECTED. The remaining foods are placed in the set PLANNED. The selection model selects the remaining foods in the 10-day cycle from the set PLANNED.

The objective function is defined as cost minimization:

$$
\boldsymbol{Z}=\boldsymbol{m i n} \sum_{j \in \text { Food }} \cos t_{j} \times x_{j}
$$

The objective function minimizes the total cost of all the foods, i.e. of the whole 10-day diet, including those chosen by the selection model and the foods chosen by the crew.

Three sets of major requirements constrained the objective function. In evaluating these constraints, the selection model considers all chosen foods in both sets (PRESELECTED and PLANNED) to ensure that the diet as a whole meets the constraints. The first set of requirements ensures that minimum and maximum nutritional requirements are met, on average, during the 10-day cycle. For each nutrient $\mathrm{i}$ in set NUTR: 


$$
\text { nutr_min }_{i} \leq\left(\sum_{j \in F o o d} \text { nutr_in_food_amt } t_{i, j} * x_{j}\right) / 10 \leq \text { nutr_max }
$$

The division by 10 converts the calculation of total nutrient to a daily average for comparison with the nutrient minimum and maximum requirements.

The second constraint requires that the average acceptability level among all the foods achieves at least a minimum level of acceptability:

$$
\sum_{j \in F o o d} \text { Acceptability_of_food }_{j} * x_{j} \geq \text { Min_acceptability } * \sum_{j \in F o o d} x_{j}
$$

The level of Min_acceptability was set at 6.5 for all scenarios. Acceptability of foods was measured on a 9-point scale (this scale varies between the category 1 , or dislike extremely and 9 , or like extremely, with 5, or neither like nor dislike as the mid-point).

The last constraint guarantees that there are enough total servings within the cycle for the required number of meals:

$$
\sum_{j \in \text { Food }} x_{j}=100
$$

Additional constraints, not shown here, ensure a balance among the individual meal or food categories, such as breakfast, lunch, dinner, soups, 
appetizers, etc. The model constraints imposed on the number of servings from each food category were derived from a prototype menu/diet (Table 2). The prototype menu was designed by the chef who developed all the recipes. The guidelines in the prototype menu design were based mostly on conventional culinary considerations and familiarity with the foods. The prototype menu can be altered if needed. The solution to the diet selection model is said to be feasible if the selected diet, including the crew selected portion, satisfies all of the constraints discussed above. It is infeasible if it violates any one of the constraints.

\section{PLEASE INSERT TABLE 2 AROUND HERE}

\section{Bias Testing and Results}

The diet selection model, was implemented using AMPL (8) software. After running the model on a basic randomly-selected set of food choices and another no-choice (i.e. fully optimized) set, the model was tested under certain scenarios biased by astronauts' possible food preferences. In the randomly selected foods scenario, twenty-five foods were randomly selected from the list of 199 available foods to create an unbiased set of choices. These were put into the PRESELECTED set of the optimization model to generate an optimized diet.. Twenty replicates of this procedure were done (with different random choices) to 
test for feasibility and cost patterns. Most people, however, do not randomly select their foods. Rather, they generally select their meals based on individual preferences for certain types of foods or ingredients. Therefore, the study required performance testing under these biased preferences. The three preferences tested were those towards desserts, high-acceptability foods, and high-sodium foods. To objectively create a bias for high acceptability foods, the list of foods was sorted based on acceptability ratings (12) and the top 25 foods were selected for the choice set.

A second set of scenarios was developed to assess the level of cost savings that can be obtained by reducing the required level of variety in the planned portion of the diet. Each of the above mentioned analyses were repeated but the maximum number of servings of non-breakfast foods in the planned portion of the diet was increased from 1 serving to 2.5 servings. Increasing the allowable number of servings per food without changing the total number of servings for all the foods included in the diet resulted in a lower level of variety. The number of servings of the chosen foods (the PRESELECTED set) was kept the same as in the first set of scenarios. The paired scenarios are identified by preference bias (random, dessert, high-acceptability, and high-sodium) and by food variety (high, low). 


\section{RESULTS AND DISCUSSION}

\section{Randomly Selected Foods}

Out of the twenty random diet problem replicates, seventeen replicates produced feasible solutions. The cost for these diet solutions averaged $16.14 \mathrm{~kg}$ $\mathrm{ESM} /$ person-day, with a standard deviation of $0.46 \mathrm{~kg} \mathrm{ESM} /$ person-day (Figure 1). When food variety was reduced, all twenty replicates became feasible (see below). The no-choice (fully optimized) diet had a cost of $15.04 \mathrm{~kg} \mathrm{ESM/person-}$

day (Figure 1), which means that the random diet cost represents approximately a $7.3 \%$ increase over the fully optimized cost in the no-choice diet.

Allowing the astronauts to choose $25-30 \%$ of the meals could give them greater control over their daily diets. However, 25-30\% was a small enough portion that the diet selection model still had enough flexibility to re-optimize the remaining $70-75 \%$ to meet the model's constraints. In addition, since the model could not re-choose foods already chosen by the astronauts, the astronauts would not be required to eat the same foods more than two or three times per cycle.

The range of nutrient levels used in all of the analyses can be viewed in Table 1. Some of the nutritional constraints were relaxed from the original levels which were based on a JSC report for the nutritional requirements of long-term 
space missions (14). The nutrient levels used in the model generally varied between a level slightly lower than the minimum recommended and a level several-fold above the maximum recommended one for each nutrient. Although a higher upper bound was allowed for many nutrients, the allowable intakes remained below toxic levels (7). In general, the diet was satisfactory for most of the nutrients with the exception of its calcium (below lower recommended intake), iron (above upper recommended intake), fiber (above upper recommended intake), and biotin contents (below lower recommended intake). Improving the nutritional quality of the diet should be possible by increasing the set of foods or by modifying the recipes of the present food set to bring the nutritional content more in line with the recommended nutritional intakes. A study of these issues was considered beyond the main focus and the scope of this paper.

\section{PLEASE INSERT TABLE 1 AROUND HERE}

\section{Highly Acceptable Foods}

One very likely bias of the astronauts would be a preference for more acceptable foods. A continuous increase in consumption of highly acceptable foods was observed during a 30-day space diet study where food consumption was allowed ad libitum (17). The cost of the high-acceptability foods diet was $15.92 \mathrm{~kg} \mathrm{ESM} /$ person-day (Figure 1), which is close to the random diet cost. 


\section{PLEASE INSERT FIGURE 1 AROUND HERE}

\section{Numerous Dessert Foods}

Due to the high preference that many people have for desserts, the model was tested for a bias toward dessert foods. The list of dessert choices was made by selecting all 20 available desserts into the choice set. Since only 20 desserts were available, the preselected set included only 20 foods instead of the 25 foods used in the other choice bias scenarios. This bias resulted in a cost of $17.26 \mathrm{~kg}$ ESM/ person-day (Figure 1), which represents a moderate increase in cost over the random and high-acceptability scenarios.

\section{High Sodium Foods}

The high-sodium bias scenario turned out to be more problematic than the first three scenarios due to the specific nutritional constraints on sodium. Initially, the high-sodium choice set was selected by sorting the list of foods based on their sodium content and choosing the top 25 for the choice set. The average daily sodium content of this set reached $2125 \mathrm{mg} /$ day, which is around $2 / 3$ of the allowable sodium in the diet. As a consequence, no feasible solution could be achieved for this set. Therefore, a sensitivity analysis was conducted to determine the level of feasibility within the high-sodium food selection. Testing 
the model on a variety of combinations within the high-sodium foods showed that a feasible solution could be achieved for any set of choices within 1,978 $\mathrm{mg}$ of sodium per day in the choice set. The resulting optimized diet had a cost of 16.85 kg ESM/ person-day (Figure 1).

For comparison purposes, the sodium content in all 20 of the randomly selected choice sets analyzed earlier was determined. The mean daily sodium content of these 20 sets was $857 \mathrm{mg}$, or an average of $34 \mathrm{mg}$ per serving of these foods. This average sodium content is less than half the content of the maximally feasible high-sodium set. Additionally, the high-acceptability and the numerousdesserts sets contained daily sodium contents of 827 and $244 \mathrm{mg}$, respectively, which is even less than the average of the random sets. Therefore, this limitation on sodium content for the high-sodium diet should not restrict the astronauts' choices to any great extent.

\section{Lower Food Variety}

As mentioned earlier, increasing the maximum allowable servings per food tends to decrease the variety of foods selected in the optimized diet. When the maximum number of servings per food was raised from 1 to 2.5 servings, all twenty of the random food sets became feasible. The cost for these random food sets averaged $14.35 \mathrm{~kg} \mathrm{ESM/person-day} \mathrm{(Figure} \mathrm{1),} \mathrm{with} \mathrm{a} \mathrm{standard} \mathrm{deviation} \mathrm{of}$ $0.28 \mathrm{~kg} \mathrm{ESM} /$ person-day. A 2-sample t-test revealed a significant difference ( $\mathrm{p}$ 
$<0.05$ ) between the costs of the high- and low-variety diets (1 serving vs. 2.5 servings). Although this difference is significant, it is not large in percentage terms. The high-acceptability diet with low variety had a cost of $14.13 \mathrm{~kg} \mathrm{ESM} /$ person-day with the 2.5 servings/food scenario which is close to the random diet cost with low variety and represents only a small reduction in cost from the highvariety case. Hence the diet is not particularly sensitive to variety. We conclude that both choice and variety can be preserved in the random and highacceptability scenarios, at little added cost.

This was not the case for the numerous-desserts diet, which had a cost of 14.65 vs. $17.26 \mathrm{~kg} \mathrm{ESM/person-day} \mathrm{in} \mathrm{the} \mathrm{1-serving/food} \mathrm{scenario.} \mathrm{This} \mathrm{result}$ shows that there is a benefit to reducing the variety level in the planned foods set when a numerous-desserts choice set is adopted. A similar finding occurs in the high-sodium case, which had a $14.25 \mathrm{~kg}$ ESM/ person-day cost vs. $16.85 \mathrm{~kg} \mathrm{ESM} /$ person-day for the high-variety and low-variety scenarios, respectively. The nochoice, low-variety diet had a cost of $13.21 \mathrm{~kg} \mathrm{ESM} /$ person-day.

Figure 1 compares the costs of the different choice biases with the two variety treatments. The random, high-acceptability and no-choice biases have comparable cost increments upon changing the number of servings/food from 1 to 2.5. However, the desserts and high-sodium foods biases have greater cost increments between the variety levels. Thus, if the choice bias carries a nutritional 
bias (e.g. high-salt foods) then providing more variety in the diet (restricting the number of servings per food) is challenged by tighter nutritional constraints, which in turn will result in a significantly higher cost diet that satisfies these constraints.

\section{CONCLUSIONS}

The diet selection model with crew choice allows an astronaut crew to provide some input into the cyclical selection of their space mission food menu. The model was found to be feasible in terms of nutritional content, acceptability, and food variety. Tests of randomly selected foods, large amounts of desserts, and highly acceptable foods all resulted in feasible solutions costing between 13.2 and $17.3 \mathrm{~kg} \mathrm{ESM} /$ person-day. Only the high-sodium food bias posed a possibly infeasible solution when taken to the extreme. However, this infeasibility can be prevented by minor restrictions on the high-sodium choices available to the crew. In addition, restricting variety in the planned portion of the diet was found to be effective in reducing cost in scenarios that had strong nutritional biases.

Future model enhancements might include implementation of inventory and labor availability constraints that could connect the diet selection process to 
the realities of food production and storage. Additionally, a user-friendly software interface that could be developed would allow the crew members to more easily input their chosen foods. Both of these changes would further enhance the model's usefulness for a real space mission.

\section{ACKNOWLEDGEMENTS}

The authors would like to thank Mr. Vardges Melkonian for his assistance and Ms. Adriana Rovers for developing the prototype menu. Support of this work by the National Aeronautics and Space Administration under NASA Grant NAG 54222 and the GE Faculty of the Future undergraduate research program is gratefully acknowledged. 


\section{BIOGRAPHICAL SKETCHES}

Carrie Vicens obtained her B.S. in Operations Research and Industrial Engineering from Cornell University in May 2000. She recently completed her MSc. in Decision Science at the London School of Economics, where her dissertation research focused on the incorporation of Bayesian reasoning into the clinical diagnostic process.

Carolyn Wang received her B.S. in Operations Research and Industrial Engineering from Cornell University (2000). During her undergraduate research, she worked under Dr. Jean Hunter and Dr. Peter Jackson to create a dynamic linear optimization process for the bioregenerative diet described in this paper. She has continued to apply the skills learned at Cornell to the consulting field as well as the consumer finance industry.

Ammar Olabi is currently a post-doctoral fellow in the Food Science Department at Cornell University. He completed his B.S. in Nutrition and Dietetics and M.S. 
in Food Science at the American University of Beirut. In August 1995 he enrolled in the Food Science and Technology Ph.D. program at Cornell University. He conducted his Ph.D. research, focused on the optimization of a bioregenerative diet, under the supervision of Dr Jean Hunter.

Peter Jackson is an associate professor in the School of Operations Research and Industrial Engineering, Cornell University. He received a B.A. in Economics from the University of Western Ontario (1975), a M.Sc. in Statistics (1978) and a Ph.D. in Operations Research (1980) from Stanford University. He conducts research in production planning, resource scheduling, and inventory management and teaches courses in industrial systems analysis, design, and engineering. He is an innovator in experiential learning and the recipient of several teaching awards.

Jean Hunter is an associate professor in Cornell's Department of Biological and Environmental Engineering. She holds a B. S. from MIT and M.S. and D. Eng. Sci. degrees from Columbia University, all in chemical engineering. Her research interests include food engineering, fermentation and enzyme technology, downstream processing, and the production of value-added products from food and agricultural residues. She has taught bioprocessing, bioseparations, a biological engineering laboratory course, and with Dr. Jackson, the undergraduate 
group research project on measurement of food preparation labor which developed the data for this paper.

\section{REFERENCES}

1. Baranski, S.; Kubiczkowa, J.; Piorko, A.; Skibniewski, F.; Bryanov, I.; Milova, E.P.; Nefedova, M.V.; Yakovleva, I.J. Electrogustometric investigations during manned space flight. Aviat Space Envir Md 54 (1): $1-5,1983$.

2. Bourland, C. T.; V. L. Kloeris. Shuttle/Mir Food Experience. $29^{\text {th }}$ ICES, SAE Technical Paper 1999-01-2016, 1999.

3. Damij, T. A. Nutrition optimization and planning system. Zbornik Biotehniske Fakultete Univerze v Ljubljani, Kmetijstvo 63: 257-268, 1994.

4. Drysdale, A.E; Hanford, A.J Advanced life support research and technology development metric-baseline, CTSD-ADV-384, JSC 39503. NASA-Johnson Space Center, November 1999.

5. Eckstein, E.F. Menu planning by computer-random approach. J Am Diet Assoc 51: 529-533, 1967.

6. Evangelos, K.L.; Hill, B.M.; Saraf, S.K.; Miller, J. Convenience food logistics model (CFLM). Nav Eng J 110: 39-50, 1998.

7. Food and Nutrition Board website. December 2001. http://www4.nas.edu/IOM/IOMHome.nsf/Pages/Food+and+Nutrition+Bo ard 
8. Fourer, R.; Gay, D.M.; Kernighan, B.W. AMPL: A Modeling Language for Mathematical Programming. Danvers: Boyd and Fraser Publishing Company; 1997.

9. Foytik, J. Very low-cost nutritious diet plans designed by linear programming. J Nutr Educ 13: 63-66, 1981.

10. Hanford, A.J. Advanced Regenerative Life Support System Study, NASA JSC 37826, Johnson Space Center, March 3, 1997.

11. Henson, S. Linear-programming analysis of constraints upon human diets. J Agr Econ 42: 380-393, 1991.

12. Hunter, J.; Olabi, A.; Spies, R.; Rovers A.; Levitsky, D. Diet design and food processing for bioregenerative life support systems. SAE 1998 Transactions. Journal of Aerospace Section I, 107: 296-307, 1999.

13. Kamen, J.M.; Peyram, D.R. Acceptability of repetitive diets. Food Technol 15: 173-177, 1961.

14. Lane, H.W.; Bourland, C.T.; Pierson, F.; Grigorov, E.; Agrueev, A.; Dobrovolsky, V. Nutritional Requirements for International Space Station. Missions up to 360 Days. JSC Report 28038. NASA Johnson Space Center, January 1996.

15. Levri, J.A.; Vaccari, D.A.; Drysdale, A.E. Theory and Application of the Equivalent System Mass Metric. International Conference On Environmental Systems, Toulouse, France. SAE Paper 2000-01-2395, July 2000.

16. Oberg, J. Red Star in Orbit. New York: Random House Inc.; 1981.

17. Olabi, A. PhD Thesis. Cornell University, Ithaca, NY; 2001.

18. Olabi, A.; Hunter, J.B.; Jackson, P.J.; Segal, M.H. et al. Work measurement videotaping technique as a means for estimating food preparation labor time of a bioregenerative diet. The $29^{\text {th }}$ International 
Conference on Environmental Systems, Denver, Colorado, Paper 199901-2075, July 12-15 1999.

19. Olabi, A.; Hunter, J.B. Cost and Quality of a bioregenerative diet. The $29^{\text {th }}$ International Conference on Environmental Systems, Denver, Colorado, Paper 1999-01-2077, July 12-15 1999.

20. Rambaut, P.C.; Smith, M.C Jr.; Leach, C.S; Whedon, G.D; Reid, J. Nutrition and responses to zero gravity. Fed Proc 36 (5): 1678-1682, 1977.

21. Sinha, P. Concepts of preference maximization in computer-assisted menu planning. J Food Process Pres 2: 75-89, 1978.

22. Sklan, D.; Dariel, I. Diet planning for humans using mixed-integer linear programming. Brit J Nutr 70: 27-35, 1993.

23. Soden, P.M.; Fletcher, L.R. Modifying diets to satisfy nutritional requirements using linear programming. Brit J Nutr 68: 565-572, 1992.

24. Stuster, J. Bold Endeavors: Lessons learned from Polar and Space Exploration. Annapolis: Naval Institute Press; 1996.

25. Ward, R.C.; Harper, J.M.; Jansen, N.B. Monte Carlo simulations of nutrient based serving sizes of food. J Food Process Pres 2: 155-174, 1978 .

26. Wheeler, R.A. Biomass Production at KSC-ALSGB: Candidate Crop Testing, 1997. http://atlas.ksc.nasa.gov/celss/plant/bpframe.htm. 
Table 1. Daily nutrient recommendations for long term space flight $\leq 360$ days (Lane et al, 1996) and nutrient ranges in optimized diets.

\begin{tabular}{|c|c|c|}
\hline Nutrient & Recommended Level & $\begin{array}{c}\text { Range in optimized } \\
\text { diets }\end{array}$ \\
\hline Calories & $\begin{array}{l}\text { Men: } 1.7(11.6 \mathrm{~W}+879) \mathrm{kcal} / \text { day } \\
\text { Women: } 1.7(8.7 \mathrm{~W}+829) \mathrm{kcal} / \text { day }\end{array}$ & $2650-3000 \mathrm{kcal}$ \\
\hline Protein & $\begin{array}{l}12-15 \% \text { of total caloric intake } \\
\text { for } 2650 \mathrm{kcal}: \quad 80-99 \mathrm{~g}\end{array}$ & $81-112 \mathrm{~g}$ \\
\hline Carbohydrate & $\begin{array}{l}50-55 \% \text { of total caloric intake } \\
\text { for } 2650 \text { kcal: } 331-364 \mathrm{~g}\end{array}$ & $338-412 \mathrm{~g}$ \\
\hline Fat & $\begin{array}{l}30-35 \% \text { of total caloric intake } \\
\text { for } 2650 \mathrm{kcal}: 88-103 \mathrm{~g}\end{array}$ & $85-117 \mathrm{~g}$ \\
\hline Total Dietary Fiber & $10-25 \mathrm{~g}$ & $<45 \mathrm{~g}^{1}$ \\
\hline Sodium & $1500-3500 \mathrm{mg}$ & $<3500 \mathrm{mg}$ \\
\hline Iron & $<10 \mathrm{mg}$ & $<35 \mathrm{mg}$ \\
\hline Calcium & $1000-1200 \mathrm{mg}$ & $>600 \mathrm{mg}$ \\
\hline Magnesium & $350 \mathrm{mg}$ & $300-560 \mathrm{mg}$ \\
\hline Vitamin A & $1000 \mu \mathrm{g}$ & $900-3500 \mu \mathrm{g}$ \\
\hline Vitamin E & $20 \mathrm{mg}$ & $18-30 \mathrm{mg}$ \\
\hline Thiamin & $1.5 \mathrm{mg}$ & $1.4-3.0 \mathrm{mg}$ \\
\hline Riboflavin & $2.0 \mathrm{mg}$ & $1.8-35 \mathrm{mg}$ \\
\hline Niacin & $20 \mathrm{mg}$ & $18-30 \mathrm{mg}$ \\
\hline Vitamin B6 & $2.0 \mathrm{mg}$ & $1.8-2.5 \mathrm{mg}$ \\
\hline Folate & $400 \mu \mathrm{g}$ & $380-600 \mu \mathrm{g}$ \\
\hline Pantothenic Acid & $5.0 \mathrm{mg}$ & $4.8-35 \mathrm{mg}$ \\
\hline Biotin & $100 \mu \mathrm{g}$ & $15-60 \mu \mathrm{g}$ \\
\hline Vitamin $\mathrm{C}$ & $100 \mathrm{mg}$ & $80-360 \mathrm{mg}$ \\
\hline
\end{tabular}

\footnotetext{
${ }^{1}$ The nutritional constraints that did not satisfy the recommended level and were considered problematic are labeled in bold.
} 
Table 2. Range of number of servings per meal or food category per 10 days

\begin{tabular}{lcc}
\hline $\begin{array}{c}\text { Meal/Food } \\
\text { Category }\end{array}$ & $\begin{array}{c}\text { Minimum } \\
\text { Servings }\end{array}$ & $\begin{array}{c}\text { Maximum } \\
\text { Servings }\end{array}$ \\
\hline Breakfast & 25 & 25 \\
Lunch Entrée & 0 & 15 \\
Dinner Entrée & 0 & 12 \\
Lunch \& Dinner & NA & $\geq 16$ \\
Entrée & & \\
& 8 & 12 \\
Side dish & 10 & 20 \\
Dessert & 2 & 7 \\
Appetizer & 8 & 12 \\
Salad & 7 & 10 \\
Soup & 4 & 7 \\
Bread & 6 & 8 \\
Beverage & 2 & 4 \\
Spread & & \\
\hline
\end{tabular}




\section{FIGURE LEGENDS}

FIGURE 1. Diet cost vs. choice bias: light=High Variety, dark=Low Variety. Bars with different letters are significantly different $(p<0.05) . \mathrm{N}=$ number of feasible solutions out of twenty random diet problem replicates. 


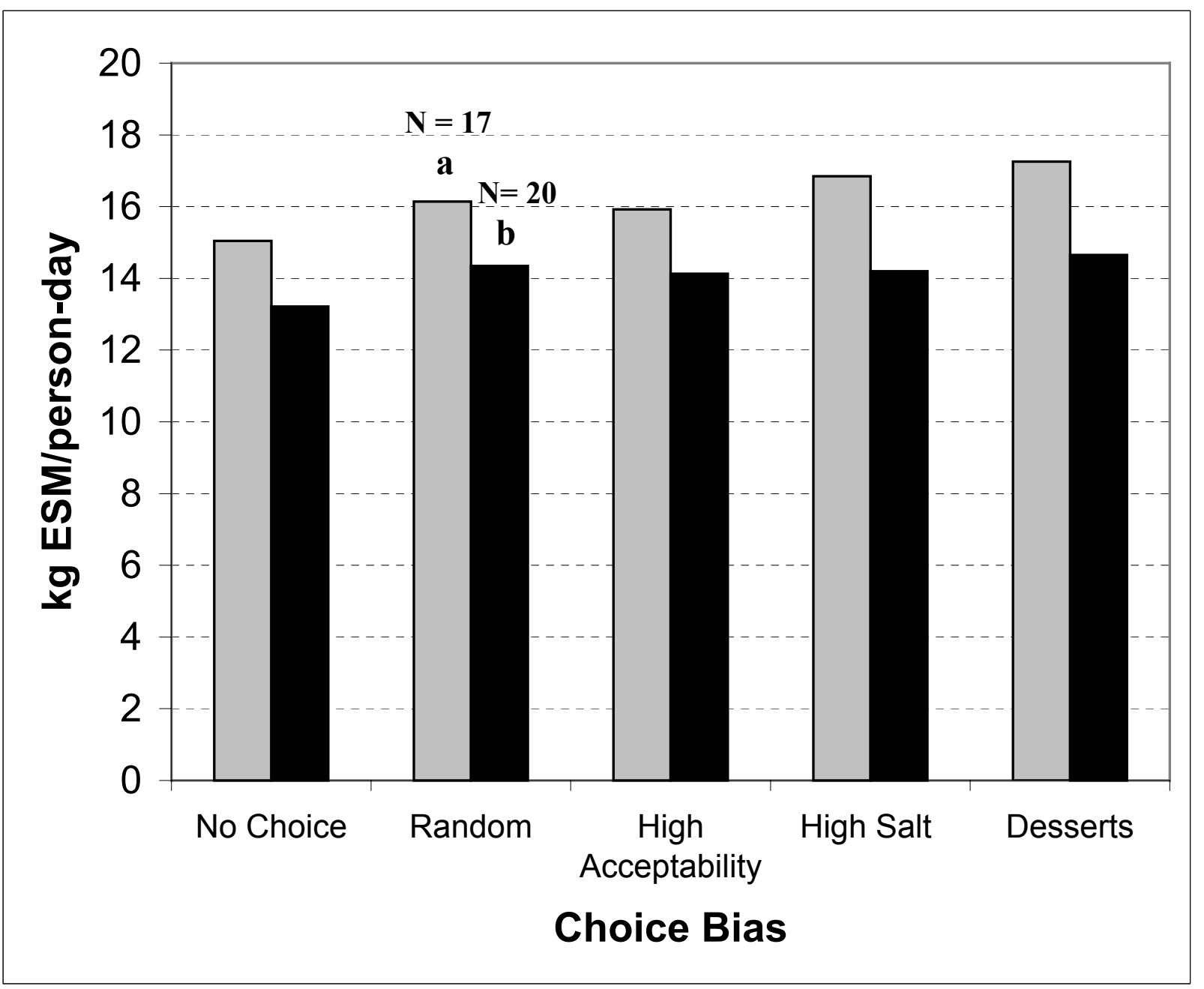

